\title{
Role of the interleukin-17 pathway in the pathogenesis of atrial fibrillation associated with inflammation
}

\author{
Honghua Yue, Jun Gu, Xueshan Zhao, Weitao Liang, Zhong Wu
}

Department of Cardiovascular Surgery, West China Hospital, Sichuan University, Chengdu, China

Submitted: 14 August 2020

Accepted: 12 November 2020

Arch Med Sci 2021; 17 (1): 262-265

DOI: https://doi.org/10.5114/aoms/130392

Copyright $\odot 2020$ Termedia \& Banach

Atrial fibrillation (AF) is the most common clinical cardiovascular disease, and one of the most important arrhythmia diseases; AF can increase the morbidity of stroke, arterial embolism and heart failure [1]. AF can result in the loss of effective atrial contraction and diastole, and lead to deterioration or loss of cardiac pumping function accompanied by irregular ventricular rhythm [2]. At present, the incidence of $A F$ in adults is about $0.4-1 \%$, and the incidence of AF in patients over 60 years old is $2-4 \%$. In addition, even without cerebral infarction, AF can cause cognitive impairment in patients, atrophy of the hippocampus, and increase the prevalence of Alzheimer's disease [3]. AF is the main cardiovascular disease that causes increased mortality and increased health care spending. In the United States, about 2.3 million people suffer from AF disease, and this number is expected to increase to 5.6 million by $2050[4,5]$. At present, the main treatment methods of AF are internal, surgical radiofrequency ablation and drug conversion therapy, however, due to the high recurrence rate of atrial fibrillation, there is no effective and safe treatment method. This is attributed to the complicated pathogenesis of AF that has not been completely clarified.

Inflammatory pathogenesis is one of the important mechanisms of atrial fibrillation. More and more evidence supports the role of inflammation in the pathophysiology of AF, which indicates that the inflammatory pathways is a potential therapeutic target, inflammation and related immune reactions are involved in the occurrence and maintenance of AF $[6,7]$. Inflammatory pathways can lead to atrial electrical and structural remodeling and thrombogenesis in patients with AF. Many inflammatory factors can cause fibrosis changes in the myocardium, which produces a substrate for the onset of AF. For instance, tumor necrosis factor (TNF) can activate transforming growth factor $\beta$ (TGF- $\beta$ ) signaling pathway and myocardial fibroblasts, increase secretion of matrix metalloproteinases-2 (MMP-2) and MMP-9, which mediate atrial remodeling [8]. In the SD (Sprague Dawley) rat model of aseptic pericarditis, interleukin-17A (IL-17A) can aggravate myocardial fibrosis and promote the occurrence of AF. Inhibition of IL-17A expression can reduce myocardial fibrosis, which shows a therapeutic effect in AF [9]. Many studies have found that IL-17A can stimulate the release of pro-inflammatory cytokines (IL-1 $\beta$, IL-6 and TGF- $\beta 1$ ), which participate in the pathogenesis of myocardial fibrosis, thereby promoting the occurrence of atrial fibrillation [10, 11].

In the study of AF-related drugs (Colchicine and Curcumin) by transcriptome [12] (Figure $1 \mathrm{~A}$, each group had three rats), we found that

\author{
Corresponding author: \\ Zhong Wu \\ Department of \\ Cardiovascular Surgery \\ West China Hospital \\ Sichuan University \\ Chengdu, China \\ Phone: +86-028-85422897 \\ E-mail: wuzhong71@163.com
}


A Transcriptome experimental workflow

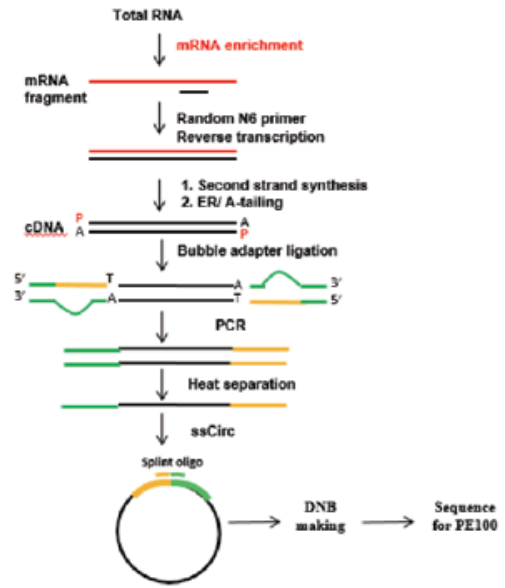

B Heatmap of hierarchical clustering of DEGs

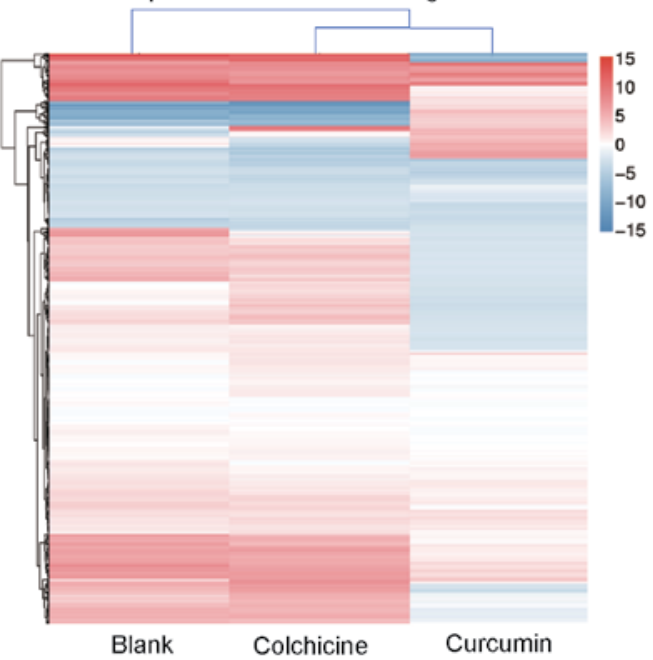

Colchicine

Curcumin

C

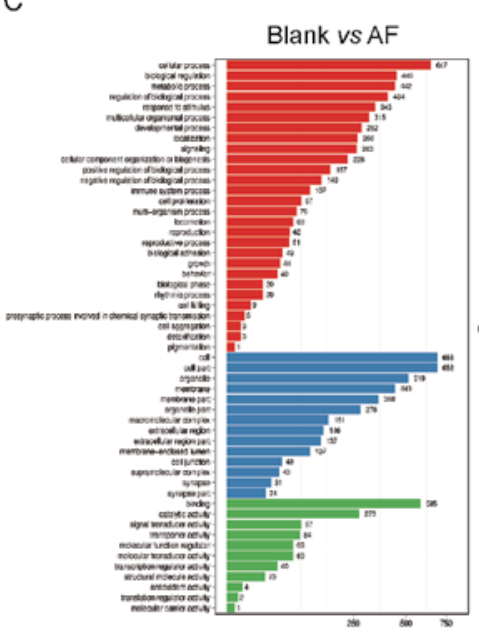

GO classification of DEGs

D

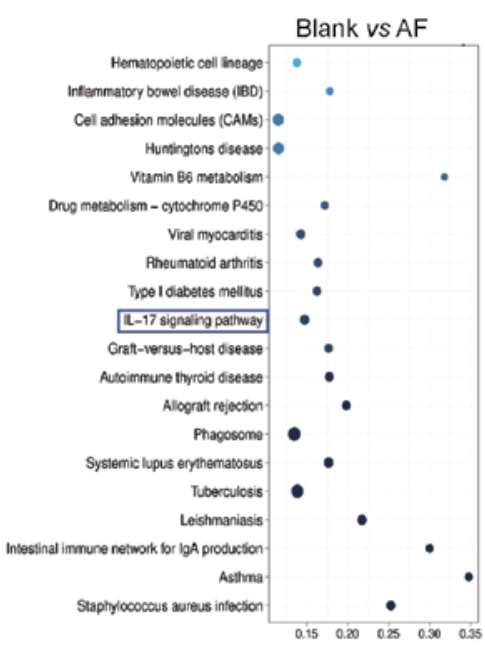

Colchicine vs AF

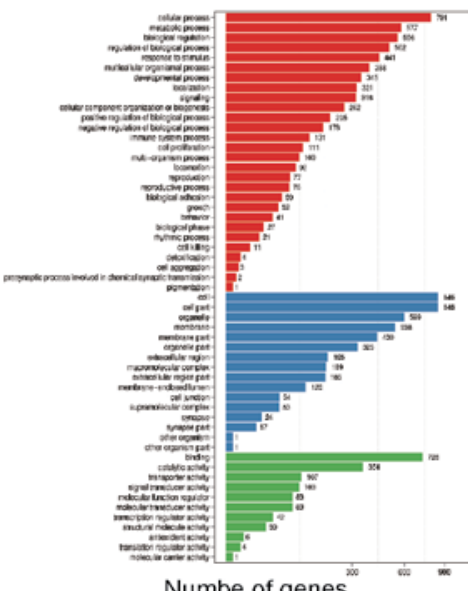

Numbe of genes

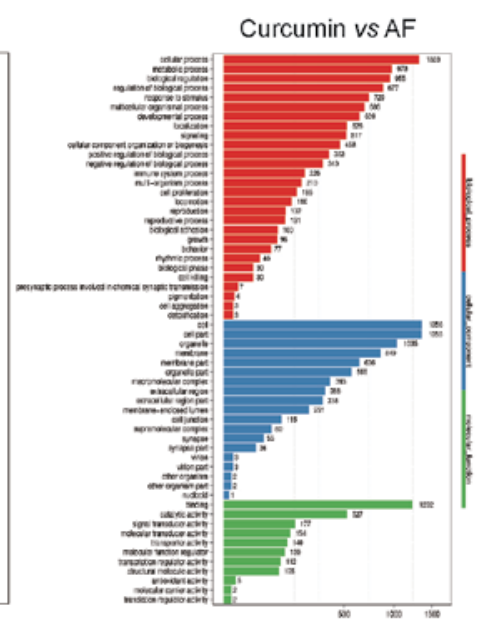

Pathway functional enrichment of DEGs
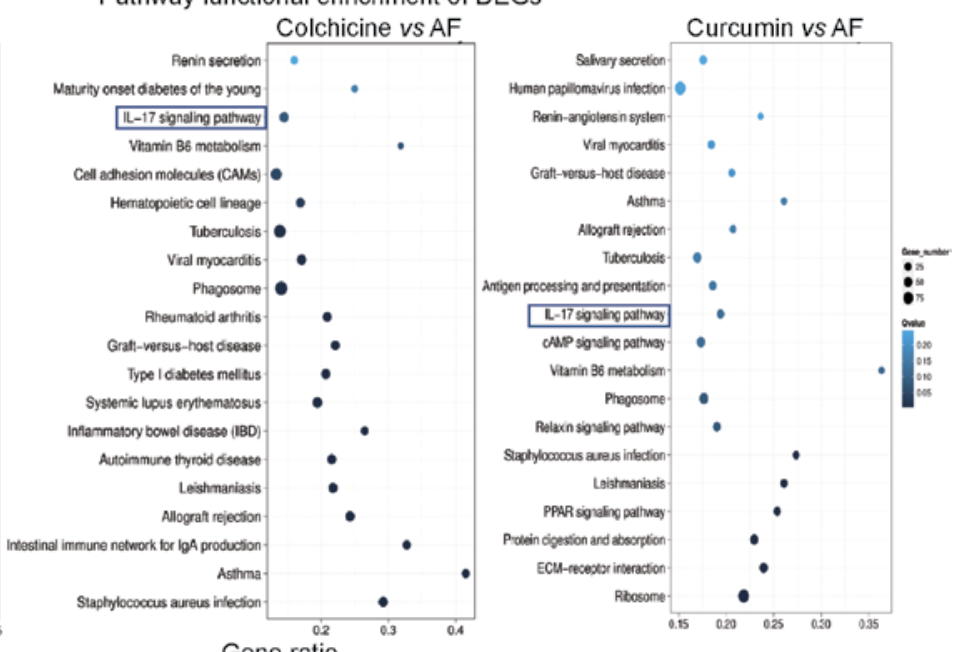

Gene ratio

Figure 1. A - Transcriptome experimental workflow. B - Heatmap of hierarchical clustering of DEGs among the blank group, AF group and Drugs treatment group ( $X$ axis represents each comparing sample. $Y$ axis represents DEGs. Colouring indicates the log2 transformed fold change. High: red, Low: blue). C - The GO classification results among the blank group, AF group and Drugs treatment group ( $X$ axis represents number of DEG. $Y$ axis represents $G O$ term). $\mathrm{D}$ - Pathway classification of DEGs among the blank group, AF group and Drugs treatment group ( $\mathrm{X}$ axis represents enrichment factor. $Y$ axis represents the pathway name. The color indicates the q-value (high: white, low: blue), the lower q-value indicates the more significant enrichment. Point size indicates DEG number (The bigger dots refer to larger amounts). Rich Factor refers to the value of the enrichment factor, which is the quotient of foreground value (the number of DEGs) and background value (total Gene amount). The larger the value, the more significant enrichment 
the IL-17 pathway is highly expressed in the AF rat model (The rat model of $\mathrm{AF}$ was induced by Ach $(66 \mu \mathrm{g} / \mathrm{ml})-\mathrm{CaCl}_{2}(50 \mathrm{mg} / \mathrm{ml})$ with a dose of $0.1 \mathrm{ml} / 100 \mathrm{~g}$ via the tail vein injection for 28 days, Atrial fibrillation model standards: the electrocardiogram showed that the $\mathrm{P}$ wave disappeared and the $f$-wave appeared as the typical atrial fibrillation ECG). The Colchicine treatment group and Curcumin treatment group were given colchicine by gavage once a day for 24 days at a therapeutic dosage of $0.8 \mathrm{mg} / \mathrm{kg}$ and $4 \mathrm{ml} / \mathrm{kg} / \mathrm{d}$ (concentration: $50 \mathrm{mg} / \mathrm{ml}$ ), and the expression of IL-17 pathway was significantly down-regulated after drug intervention (Figure 1 D) [12]. The emerging role of IL-17 as a hallmark proinflammatory cytokine of the adaptive immune system produced by a new T helper cell subset termed "Th17" has received considerable attention. IL-17 is an important pro-inflammatory cytokine that plays an important role in the innate and acquired immune systems of the body. The IL-17 family can promote the body to resist infection of various pathogens, and is closely related to the occurrence and development of autoimmune diseases, tumors and other diseases [13]. The family consists of six members: IL-17A, IL-17B, IL-17C, IL-17D, IL-17E (also known as IL-25) and IL-17F. Among them, IL-17A and IL-17F play an important role in Th17 ( $T$ helper cell 17 ) cellular immunity. IL-17A and IL-17F are $50 \%$ homologous and can form either homologous dimers or heterodimers that bind to IL-17RA/IL-17RC, which can activate downstream nuclear factor $\kappa \mathrm{B}(\mathrm{NF}-\kappa \mathrm{B})$ and mitogen-activated protein kinase (MAPK) signaling pathways by acting on signaling complex IL-17R-ACT1-TRAF6 [14]. The IL-17 pathway participates in the body's anti-infection immunity, pathological inflammatory response related to autoimmune diseases, and the occurrence and progression of tumors.

Comprehensive genome-wide screening of the transcriptome has shown the mechanism of dis- ease differentiation and possible diagnostic and treatment value. Analysis of the expression profiles among the Blank group, AF group and Drugs treatment group and the profound analysis of Differentially Expressed Genes (DEGs) changes may contribute to the comprehensive understanding of the pathology of AF and potential therapeutic mechanisms. Hierarchical clustering analysis revealed that the genomic expression of three groups of tissues had differences in transcriptomics profiles (Figure 1 B). Genes, such as IL17a, IL17b, Mapk13, Mapk15, Lcn2, Cxcl10, Rsph10b, Ccl11, Irx3 and $\mathrm{Ccl} 20$ are included in the IL-17 pathway (Table I). Moreover, these genes were highly expressed in the AF group and significantly down-regulated after drug intervention, indicating that these genes can be used as potential therapeutic targets in IL-17 pathway. In addition, we perform the Gene Ontology (GO) classification and functional enrichment with DEGs. GO has three ontologies: molecular biological function, cellular component and biological process, most of them participated in the cell process, cell part, binding, etc (Figure $1 \mathrm{C}$ ).

In conclusion, we provide an integrative analysis of transcriptomics for studying the pathogenesis of AF. We first discovered that the IL-17 signaling pathway plays an important role in the pathogenesis of AF and that some genes can be used as potential therapeutic targets for AF. Due to the importance of the IL-17 pathway in the pathology in this study, targeting differential genes in the IL-17 pathway may become new diagnostic and therapeutic methods in the future.

\section{Acknowledgments}

The RNAseq data from this study have been submitted and archived to the NCBI Sequence Read Archive under sample accession number SRP162107 and SRP189088.

This study was funded by the National Natural Science Foundation of China (grant number

Table I. Details of key genes in the combination of DEGs in the IL-17 pathway

\begin{tabular}{|lccccc|}
\hline Gene ID & Variation type & Symbol & $\begin{array}{l}\text { Kegg Orthology } \\
\text { (IL-17 pathway) }\end{array}$ & Fold change & $P$-value \\
\hline 301289 & Up-to-down & IL17a & Ko04657 & 4.56870 & 0.001049 \\
\hline 116472 & Up-to-down & IL17b & Ko04657 & 1.52430 & $1.818 \mathrm{E}-09$ \\
\hline 29513 & Up-to-down & Mapk13 & Ko04657 & 2.51562 & $1.618 \mathrm{E}-34$ \\
\hline 286997 & Up-to-down & Mapk15 & Ko04657 & 5.54561 & $1.059 \mathrm{E}-68$ \\
\hline 170496 & Up-to-down & Lcn2 & Ko04657 & 1.40723 & $2.854 \mathrm{E}-114$ \\
\hline 245920 & Up-to-down & Cxcl10 & Ko04657 & 1.10146 & $1.436 \mathrm{E}-21$ \\
\hline 288478 & Up-to-down & Rsph10b & Ko04657 & 5.55190 & $2.333 \mathrm{E}-233$ \\
\hline 29397 & Up-to-down & Ccl11 & Ko04657 & 1.28576 & $3.759 \mathrm{E}-10$ \\
\hline 307721 & Up-to-down & Irx3 & Ko04657 & 1.39877 & $1.443 \mathrm{E}-12$ \\
\hline 29538 & Up-to-down & Ccl20 & Ko04657 & 3.81381 & $5.056 \mathrm{E}-09$ \\
\hline
\end{tabular}

These genes were highly expressed in the AF group and significantly down-regulated after drug intervention. 
81671777, 81700410); Post-Doctor Research Project, West China Hospital, Sichuan University, and funded by the Department of Science and Technology of Sichuan Province (grant number 2019YFS0344). We also gratefully acknowledge the support from many collaborators for their help in RNAseq experiments and also BGI Genomics Company for sequencing services they provided to us.

Honghua Yue, Jun Gu and Xueshan Zhao contributed equally to this article.

\section{Conflict of interest}

The authors declare no conflict of interest.

\section{References}

1. Wańkowicz P, Nowacki P, Gołąb-Janowska M. Risk factors for ischemic stroke in patients with non-valvular atrial fibrillation and therapeutic international normalized ratio range. Arch Med Sci 2019; 15: 1217-22.

2. January CT, Wann LS, Alpert JS, et al. 2014 AHA/ACC/HRS guideline for the management of patients with atrial fibrillation: executive summary: a report of the American College of Cardiology/American Heart Association Task Force on practice guidelines and the Heart Rhythm Society. Circulation 2014; 130: 2071-104.

3. Staerk L, Sherer JA, Ko D, Benjamin EJ, Helm RH. Atrial fibrillation: epidemiology, pathophysiology, and clinical outcomes. Circ Res 2017; 120: 1501-17.

4. Kannel WB, Benjamin EJ. Current perceptions of the epidemiology of atrial fibrillation. Cardiol Clin 2009; 27: 13-24.

5. Zimetbaum P. Atrial fibrillation. Ann Intern Med 2017; 166: Itc33-48.

6. Guo Y, Lip GY, Apostolakis S. Inflammation in atrial fibrillation. J Am Coll Cardiol 2012; 60: 2263-70.

7. Hu YF, Chen YJ, Lin YJ, Chen SA. Inflammation and the pathogenesis of atrial fibrillation. Nat Rev Cardiol 2015; 12: 230-43.

8. Liew R, Khairunnisa K, Gu Y, et al. Role of tumor necrosis factor-alpha in the pathogenesis of atrial fibrosis and development of an arrhythmogenic substrate. Circ J 2013; 77: 1171-9.

9. Fu XX, Zhao N, Dong Q, et al. Interleukin-17A contributes to the development of post-operative atrial fibrillation by regulating inflammation and fibrosis in rats with sterile pericarditis. Int J Mol Med 2015; 36: 83-92.

10. Valente AJ, Yoshida T, Gardner JD, Somanna N, Delafontaine P, Chandrasekar B. Interleukin-17A stimulates cardiac fibroblast proliferation and migration via negative regulation of the dual-specificity phosphatase MKP-1/ DUSP-1. Cell Signal 2012; 24: 560-8.

11. Onishi RM, Gaffen SL. Interleukin-17 and its target genes: mechanisms of interleukin-17 function in disease. Immunology 2010; 129: 311-21.

12. Yue H, Liang W, Gu J, et al. Comparative transcriptome analysis to elucidate the therapeutic mechanism of colchicine against atrial fibrillation. Biomed Pharmacother 2019; 119: 109422.

13. Murdaca G, Colombo BM, Puppo F. The role of Th17 lymphocytes in the autoimmune and chronic inflammatory diseases. Intern Emerg Med 2011; 6: 487-95.

14. Miossec P, Kolls JK. Targeting IL-17 and Th17 cells in chronic inflammation. Nat Rev Drug Discov 2012; 11: 763-76. 